\title{
Bent It Like FRs: Extended Radio AGN in the COSMOS Field and Their Large-Scale Environment
}

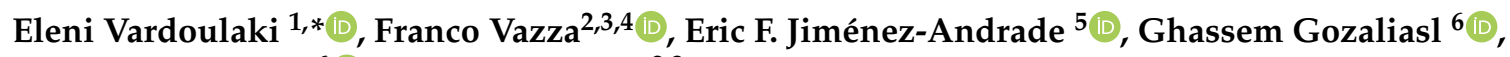 \\ Alexis Finoguenov ${ }^{6}$ and Denis Wittor ${ }^{2,3}$ \\ 1 Thüringer Landessternwarte, Sternwarte 5, 07778 Tautenburg, Germany \\ 2 Dipartimento di Fisica e Astronomia, Universita di Bologna, Via Gobetti 93/2, 40122 Bologna, Italy; \\ franco.vazza2@unibo.it (F.V.); dwittor@hs.uni-hamburg.de (D.W.) \\ 3 Hamburger Sternwarte, Gojenbergsweg 112, 21029 Hamburg, Germany \\ 4 Istituto di Radioastronomia, INAF, Via Gobetti 101, 40122 Bologna, Italy \\ 5 National Radio Astronomy Observatory, 520 Edgemont Road, Charlottesville, VA 22903, USA; \\ ejimenez@nrao.edu \\ 6 Department of Physics, University of Helsinki, P. O. Box 64, FI-00014 Helsinki, Finland; \\ ghassem.gozaliası@elsinki.fi (G.G.); alexis.finoguenov@helsinki.fi (A.F.) \\ * Correspondence: elenivard@gmail.com
}

Citation: Vardoulaki, E.; Vazza, F.; Jiménez-Andrade, E.F.; Gozaliasl, G.; Finoguenov, A.; Wittor, D. Bent It Like FRs: Extended Radio AGN in the COSMOS Field and Their Large-Scale Environment. Galaxies 2021, 9, 93. https://doi.org/10.3390/ galaxies 9040093

Academic Editor: Wenwu Tian

Received: 29 September 2021

Accepted: 2 November 2021

Published: 5 November 2021

Publisher's Note: MDPI stays neutral with regard to jurisdictional claims in published maps and institutional affiliations.

Copyright: (c) 2021 by the authors. Licensee MDPI, Basel, Switzerland. This article is an open access article distributed under the terms and conditions of the Creative Commons Attribution (CC BY) license (https:// creativecommons.org/licenses/by/ $4.0 /)$.

\begin{abstract}
A fascinating topic in radio astronomy is how to associate the complexity of observed radio structures with their environment in order to understand their interplay and the reason for the plethora of radio structures found in surveys. In this project, we explore the distortion of the radio structure of Fanaroff-Riley (FR)-type radio sources in the VLA-COSMOS Large Project at $3 \mathrm{GHz}$ and relate it to their large-scale environment. We quantify the distortion by using the angle formed between the jets/lobes of two-sided FRs, namely bent angle (BA). Our sample includes 108 objects in the redshift range $0.08<z<3$, which we cross-correlate to a wide range of large-scale environments (X-ray galaxy groups, density fields, and cosmic web probes) in the COSMOS field. The median BA of FRs in COSMOS at $z_{\text {med }} \sim 0.9$ is $167.5_{-37.5}^{+11.5}$ degrees. We do not find significant correlations between BA and large-scale environments within COSMOS covering scales from a few kpc to several hundred Mpc, nor between BA and host properties. Finally, we compare our observational data to magnetohydrodynamical (MHD) adaptive-mesh simulations ENZO-MHD of two FR sources at $z=0.5$ and at $z=1$. Although the scatter in BA of the observed data is large, we see an agreement between observations and simulations in the bent angles of FRs, following a mild redshift evolution with BA. We conclude that, for a given object, the dominant mechanism affecting the radio structures of FRs could be the evolution of the ambient medium, where higher densities of the intergalactic medium at lower redshifts as probed by our study allow more space for jet interactions.
\end{abstract}

Keywords: radio sources; active galaxies; galaxy groups; clusters; environment; radio; X-rays; simulations

\section{Introduction}

Extended radio active galactic nuclei (AGN) often deviate from a straight radio structure, and their shapes present a plethora of distortions. Past and current radio surveys add on the complexity of radio structures, especially high-sensitivity and high-resolution surveys since they reveal features in the radio structure not seen with past surveys. The reason behind bent radio structures is likely to be a complex phenomenon. Relative motions of the source or of the jets through a dense intergalactic (IGM) or intracluster medium (ICM), as well as interaction with nearby sources, can cause deviations from the expected straight radio structure, e.g., [1-8]. Thus, the role of the large-scale environment is considered crucial in shaping extended radio AGN. Recent studies show links between richness of cluster environment and bent angle [8]. It has also been shown that bent radio sources prefer cluster environments, e.g., [9] and that when FRI or FRII jets expand into denser environments the expansion is suppressed [7]. Given 
that radio sources can be found in different types of cluster/group environments, the degree of complexity could vary from source to source in relation to its small-/large-scale environment. Thus, performing a study of different types of environments can help us identify the reason why extended radio AGN get their jets bent.

In this study, we examine the degree of distortion of radio AGN in the COSMOS field [10] in order to take advantage of the plethora of multi-wavelength data and environmental probes. We quantify this distortion by the angle jets/lobes form to each other, namely bent angle (BA). We compare to large-scale environments in COSMOS and in particular to the X-ray galaxy groups studied by Gozaliasl et al. [11], the density fields the large-scale structure studied by Scoville et al. [12], and the cosmic-web probes studied by Darvish et al. [13,14]. To our knowledge, our study of BA and its relation with galaxy groups is the first one of its kind. The redshift range we cover is $0.08<z<3$, and we examine environmental probes from a few kpc to $500 \mathrm{Mpc}$. Finally, we compare our results with the evolution of two simulated radio sources at redshifts $z=0.5$ and $z=1$, generated by magnetohydrodynamical (MHD) adaptive-mesh simulations ENZO-MHD by Vazza et al. [15].

\section{Sample}

The sample is drawn from Vardoulaki et al. [16], who classified the extended radio AGN at 3 GHz VLA-COSMOS based on the classification scheme of Fanaroff and Riley [17] as FRII or edge-brightened, FRI or edge darkened, and added to that a classification of hybrid FRI/FRII objects; the latter have a jet on one side and a lobe on the other. We deem objects that fall into these classes FR-type radio sources throughout the paper. From the 130 FRs reported in [16], we use 108 for the current analysis where bent angles (BA) are available. These were measured through visual inspection of the projected sources, which could add a source of uncertainty. The values can be found in [16]. The BA is given in degrees and is the angle formed between jets/lobes with respect to each other in a twosided source (Figure 1). The Vardoulaki et al. [16] sample also includes jet-less radio AGN and star-forming galaxies, which we do not include in this analysis due to their jet-less radio structure.

\section{straight: $180^{\circ}$}
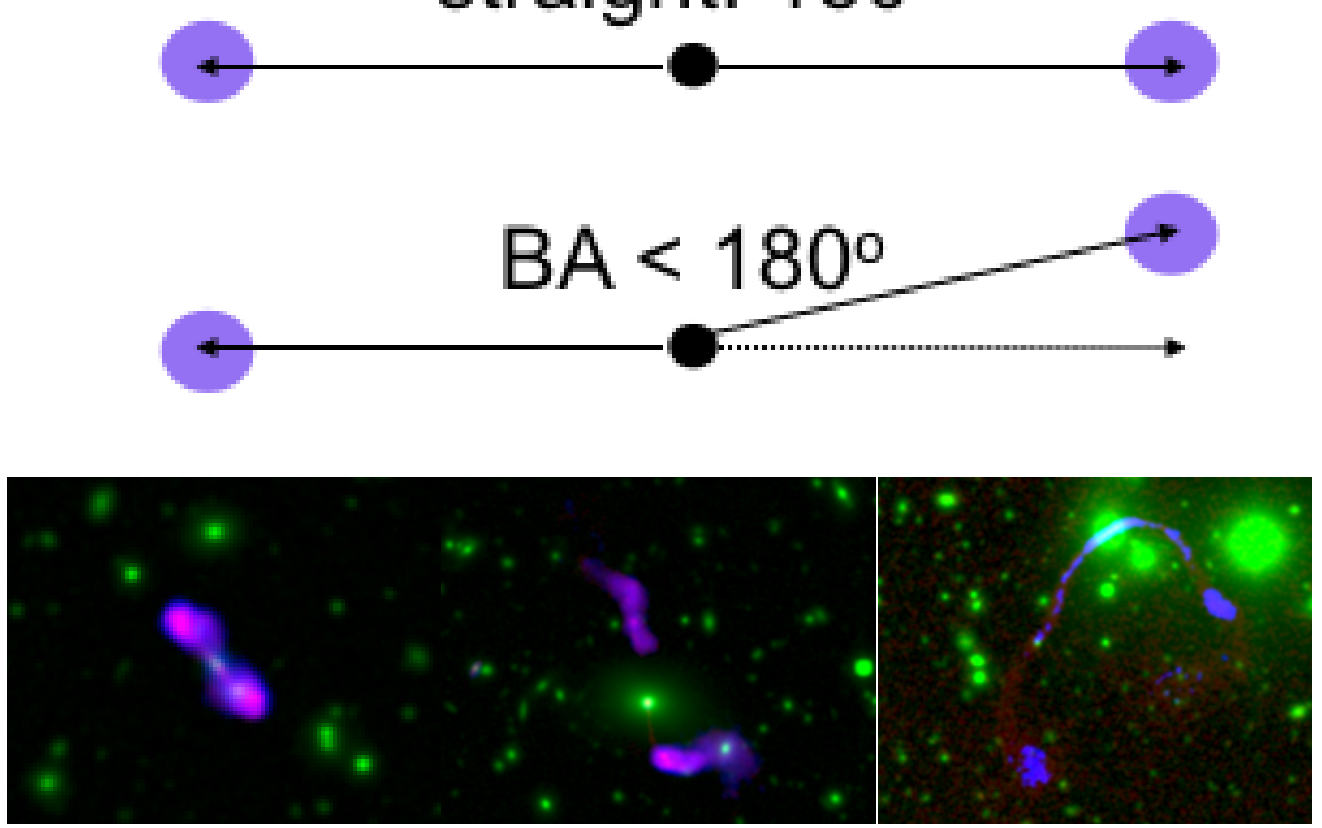

Figure 1. Top: Sketch showing how the bent angle was measured. Bottom: Examples of bent FRs from [16] , for $\mathrm{BA}=180^{\circ}$ (left), $139^{\circ}$ (middle), and $57^{\circ}$ (right). The radio emission at 1.4 and $3 \mathrm{GHz}$ is shown in purple, and the infrared Ultra-VISTA stacked mosaic [18] with green. The latter identifies the host of the radio AGN. 


\section{Analysis and Results}

\subsection{Bent Angle vs. FR Type}

The median values of the 108 FRs with BA are listed in Table 1. The FR population at $3 \mathrm{GHz}$ VLA-COSMOS has a median $\mathrm{BA}=167.5 \pm 16.1$ degrees, with minimum $\mathrm{BA}=37$ degrees and maximum $\mathrm{BA}=180$ degrees. The latter value represents objects in which their jets/lobes are on a straight line (see Figure 1). The median redshift of the FR population presented here is $z_{\text {med }}=0.9$. In Figure 2, we show the redshift range covered by our sample in relation to the BA of the objects. In Table 1, we also provide the median values for the different FR types. There is a large scatter in the values and an overlap in the distributions. Based on the Kolmogorov-Smirnov test for goodness of fit (K-S statistic and its $p$-value), we cannot reject the null hypothesis that the distributions of FRI and FRII (K-S statistic $=0.16, p$-value $=0.66)$ and FRI $/$ FRII and FRII $(\mathrm{K}-\mathrm{S}$ statistic $=0.18, p$-value $=0.49)$ are identical. In Figure 2, we also show the cumulative distributions for the three FR types, which shows that FRIs reach smaller bent angles (i.e., more bent radio lobes) than FRIIs and FRI/FRIIs.


Figure 2. (Left): Bent angle in degrees of COSMOS FR sources at $3 \mathrm{GHz}$ versus redshift. For reference, the large-scale environments we use in the analysis cover the redshift range up to $z \sim 3$, depending on the probe. (Right): Cumulative distributions of BA for the different FR populations, with FRIIs in red, FRI/FRIIs in green, and FRIs in blue.

Table 1. Median bent angle of FRs sources in our sample. The error on the median is $\sim 1.253 \times \sigma / \sqrt{(} N)$, assuming a normal distribution.

\begin{tabular}{lccccc}
\hline $\begin{array}{l}\text { Radio } \\
\text { Class }\end{array}$ & $\boldsymbol{N}$ & Median \pm Error & $\begin{array}{c}\text { Bent Angle (deg.) } \\
\text { Median }_{\mathbf{1 6} \%}^{84 \%}\end{array}$ & Min & Max \\
\hline FRII & 59 & $170.0 \pm 4.25$ & $170.00_{140.1}^{179.0}$ & 57.0 & 180.0 \\
FRI/FRII & 25 & $165.0 \pm 6.30$ & $165.00_{132.0}^{175.3}$ & 101.0 & 180.0 \\
FRI & 24 & $167.0 \pm 9.29$ & 167.0180 .0 & 37.0 & 180.0 \\
\hline
\end{tabular}

\subsection{Bent Angle vs. Large-Scale Environment and Galaxy Type}

We firstly investigate the BA of FRs in relation to large-scale environments of the order of Mpc scales. We cross-correlate the FR sample to the density fields in COSMOS [12] to infer relations to BA. In particular, we make use of the density per co-moving $\mathrm{Mpc}^{2}$, 
the large-scale structure in COSMOS, calculated using adaptive smoothing and Voronoi tessellation [12] using extremely accurate photometric redshifts available for COSMOS. The density fields are given in a cube of redshift slices ${ }^{1}$ up to redshift of 3 , which includes all the objects in our sample. In Figure 3, we plot the bent angle parameter for our FRs as a function of density per $\mathrm{Mpc}^{2}$. Most of the FRs in our sample lie in low-density environments, with mean density values of $0.84 \pm 0.11 \mathrm{Mpc}^{-2}$ for FRIIs, $0.95 \pm 0.18 \mathrm{Mpc}^{-2}$ for FRI/FRIIs, and $0.59 \pm 0.10 \mathrm{Mpc}^{-2}$ for FRIs. We do not find a correlation between BA and COSMOS density fields. Objects with small BA $\left(<100^{\circ}\right)$ can be found in similar surface density environments to the ones above that angle, namely with densities of $\sim 10 \mathrm{per} \mathrm{Mpc}^{2}$. We do not find objects at $3 \mathrm{GHz}$ VLA-COSMOS with small BA $\left(<75^{\circ}\right)$ in less dense environments $\left(<10 \mathrm{Mpc}^{-2}\right)$. Additionally, we do not find objects with $\mathrm{BA}<130$ degrees in densities below $<0.1 \mathrm{Mpc}^{-2}$. While the latter suggests that the radio structure of the sources in our sample is less affected in less dense environments, we note that a K-S statistics does not reveal a statistically significant difference between the parent distribution of objects residing in densities below and above $<0.1 \mathrm{Mpc}^{-2}$, partially due to the poor sampling of our study at such low densities.
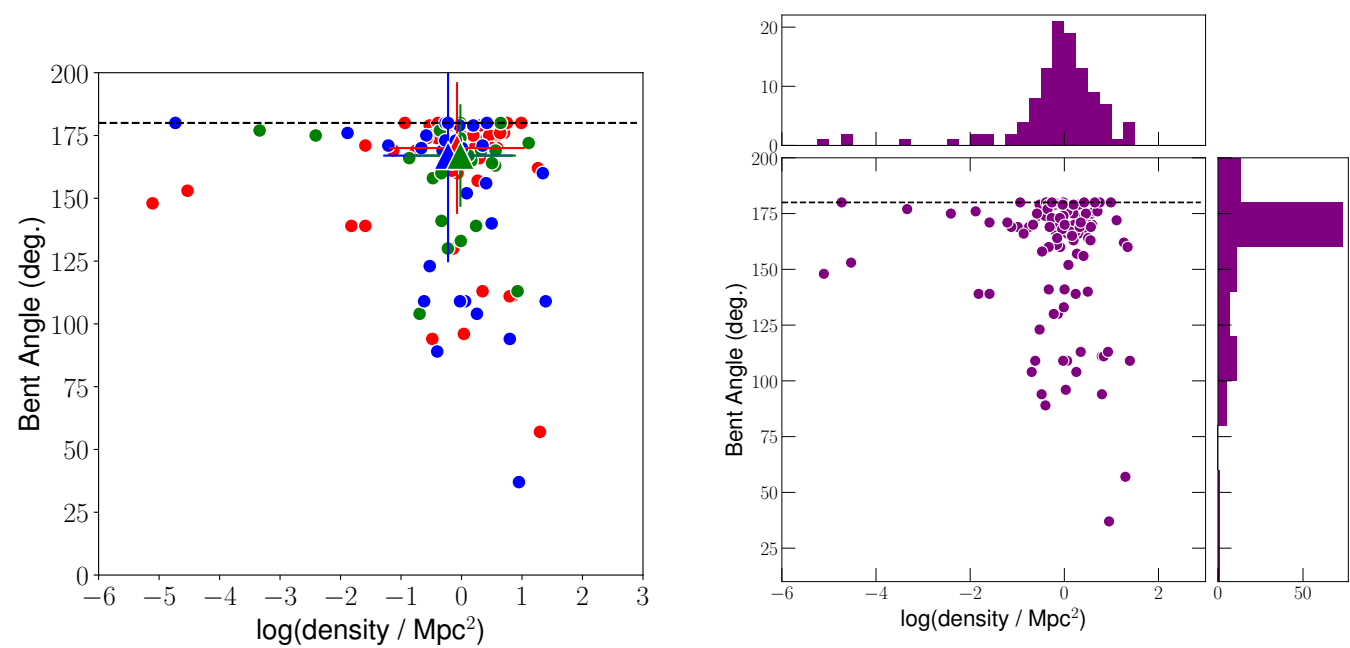

Figure 3. (Left): Bent angles in degrees for FRIIs (red), FRI/FRIIs (green), and FRIs (blue) as a function of density per co-moving $\mathrm{Mpc}^{2}$ from [12]. Large triangles are the mean values for FRIIs (red), for FRI/FRIIs (green), and for FRIs (blue), while the error bar gives the standard deviation. (Right): Same plot as on the left panel, with all FRs plotted as purple circles. In both panels, the horizontal dashed line marks a straight radio source where the jets or lobes form a $180^{\circ}$ angle.

In Figure 4, we compare the median bent angle of the FRII, FRI/FRII, and FRI objects in our sample to the large-scale environments as characterized by the study of [14] up to redshift of 1.2, by making use of the density fields in COSMOS from [12,13]. Darvish et al. [14] characterized the cosmic web environment as cluster, filament, or field, and host environment as central, satellite, or isolated. Isolated hosts, based on the definition of Darvish et al., are sources not associated with galaxy groups. These can be centrals whose satellites are too faint to detect in the volume-limited sample or they are satellites ejected beyond the virial radius. In Table 2, we list the number of FR objects in each cosmic web environment.

In Figure 4, we do not observe a clear relation between BA and cosmic web probes, probably due to the small-number statistics (Table 2). Only $\sim 44 \%$ of our FR sample is associated with the cosmic web probes characterised by Darvish et al., and mostly sources with BA $>150$ degrees. From Figure 4, we see an indication that FRI/FRIIs in filaments are more bent than FRIs and FRIIs. Similarly, FRIs in satellite hosts and FRI/FRIIs in isolated hosts are more bent in filaments, with median BA below 150 degrees, but the small number of sources affects our interpretation. 
Table 2. FRs with BA measurements in cosmic web probes. Cross-correlation of the FR and COM AGN samples with [14] with respect to different environments (cluster, filament, and field). In the parentheses, we give the percentage over the total cross-matched number of objects with the same type (within these environments).

\begin{tabular}{cccc}
\hline Radio Class & Cluster & Filament & Field \\
\hline FRII & $10(46 \%)$ & $6(27 \%)$ & $6(27 \%)$ \\
FRI/FRII & $4(36 \%)$ & $4(36 \%)$ & $3(28 \%)$ \\
FRI & $4(27 \%)$ & $8(53 \%)$ & $3(20 \%)$ \\
\hline
\end{tabular}
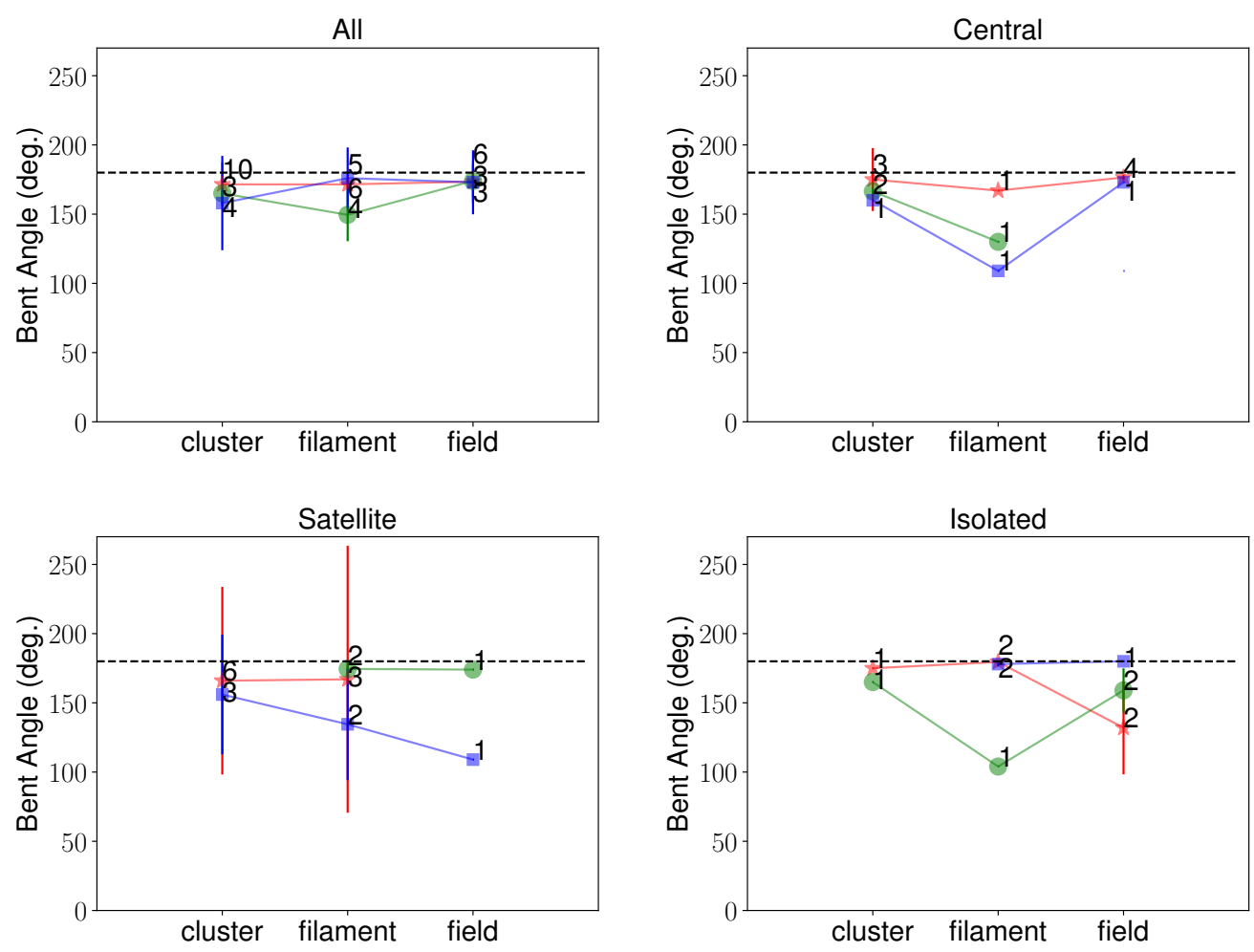

Figure 4. Bent angle in degrees for FRIIs (red), FRI/FRIIs (green), and FRIs (blue) as a function of the environment (cluster, filament, and field) depending on galaxy type (central, satellite, isolated), as defined in [14]. The horizontal dashed line marks a straight radio source where the jets or lobes form a $180^{\circ}$ angle. The numbers next to the symbols show the number of objects in that bin. The errors are the errors of the median $(\sim 1.253 \sigma / \sqrt{N}$, assuming a normal distribution).

\subsection{Bent-Angle vs. X-ray Galaxy Group Properties}

The analysis of BA and large-scale environments within COSMOS did not reveal any obvious correlation with BA. Thus, we investigate possible correlations on smaller scales (kpc to $2 \mathrm{Mpc}$ ), within X-ray galaxy groups, identified using Chandra and XMM observations of the COSMOS field [11]. We cross-correlate the FRs to the X-ray galaxy groups [11] that cover the redshift range up to $z=1.5$. In Figure 5 (Left) we plot the bent angle as a function of the distance from the $\mathrm{X}$-ray galaxy group center [11], normalized to the virial radius $r_{200}$ of each group. We mark the brightest galaxy in the group, BGG, with a star. We do not find a relation between the FR being associated with BGGs and the location of the host with respect to the X-ray galaxy group center. From the 13 FRs with straight radio structure (BA $=180$ degrees), only 2 are members of $X$-ray galaxy groups.

For objects inside $\mathrm{X}$-ray groups, we have $\mathrm{BA}_{\text {med }}=168.5 \pm 8.87$ degrees, while outside the $\mathrm{X}$-ray groups $\mathrm{BA}_{\text {med }}=168.0 \pm 4.04$ degrees. Beyond the redshift coverage of the $\mathrm{X}$-ray galaxy groups, i.e., for $z>1.5$, we have $\mathrm{BA}_{\text {med }}=167.0 \pm 5.27$ degrees. On average, there is 
no difference in the BA of FRs inside X-ray galaxy groups and outside X-ray galaxy groups.
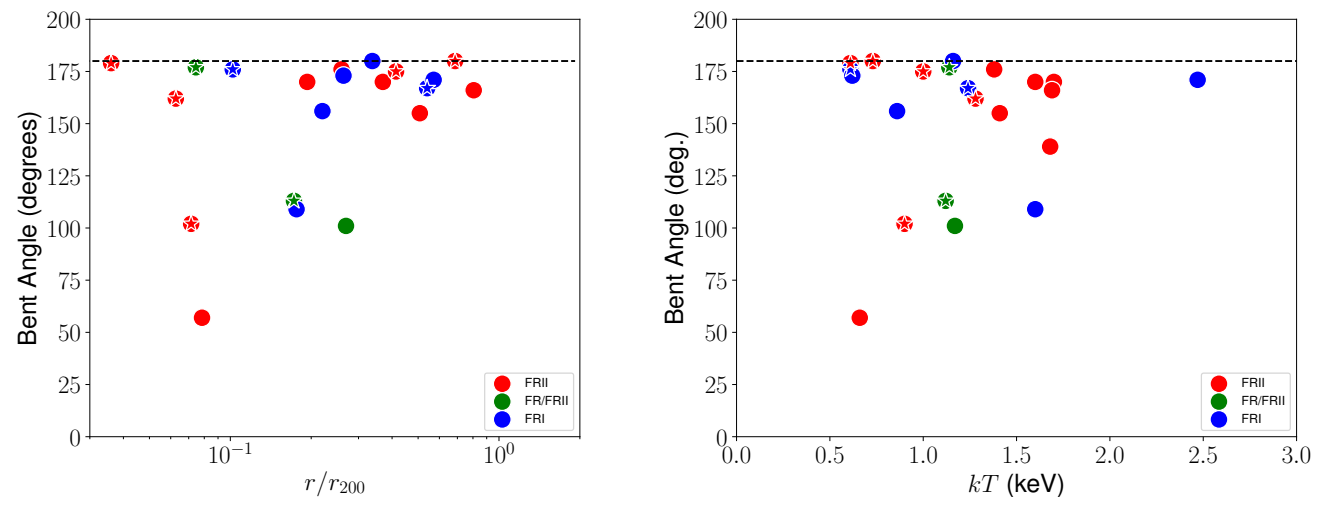

Figure 5. Bent angle in degrees for FRIIs (red), FRI/FRIIs (green), and FRIs (blue) as a function of the distance from the $\mathrm{X}$-ray group center normalized by the virial radius $r_{200}$ of the group (left) and as a function of the group temperature $k T$ in $\mathrm{keV}$ of the $\mathrm{X}$-ray galaxy group (right). The horizontal dashed line marks a straight radio source where the jets or lobes form a $180^{\circ}$ angle.

In Figure 5 (Right), we plot the bent angle as a function of the temperature $k T$ $(0.5<\mathrm{keV}<2)$ of the X-ray galaxy group [11]. There is one FRI, source 773, with $k T \sim 2.5 \mathrm{keV}$. We do not find any strong correlation between bent-angle and X-ray group temperature. This is probably related to the fact that $\mathrm{X}$-ray galaxy groups in COSMOS are not yet dynamically relaxed, which was mentioned in the analysis of [16] of FR radio source properties vs. X-ray galaxy group properties.

Additionally, in Figure 6, we compare the BA to the mass of the X-ray groups, $M_{200}$, and to the stellar mass of the host galaxy, $M_{*}$. There is no trend between $\mathrm{X}$-ray group mass nor host stellar mass and the BA. Furthermore, there is no significant difference in the BA of BGGs and non-BGGs regarding the mass of the group and the distance of the FR from the group center. This can be seen in Figure 6 on the left panel, where the size symbol increases with distance from the X-ray group center. It can also be seen with the host stellar mass on the right panel of Figure 6.

Although at smaller scales, within galaxy groups, there are no obvious correlations between BA and group properties, we find that the BA is always large (close to 180 degrees) for sources at a large distance from the X-ray group center and high temperature of the $\mathrm{X}$-ray group. The BA of radio galaxies closer to the $\mathrm{X}$-ray group center and with low temperature shows a large scatter.
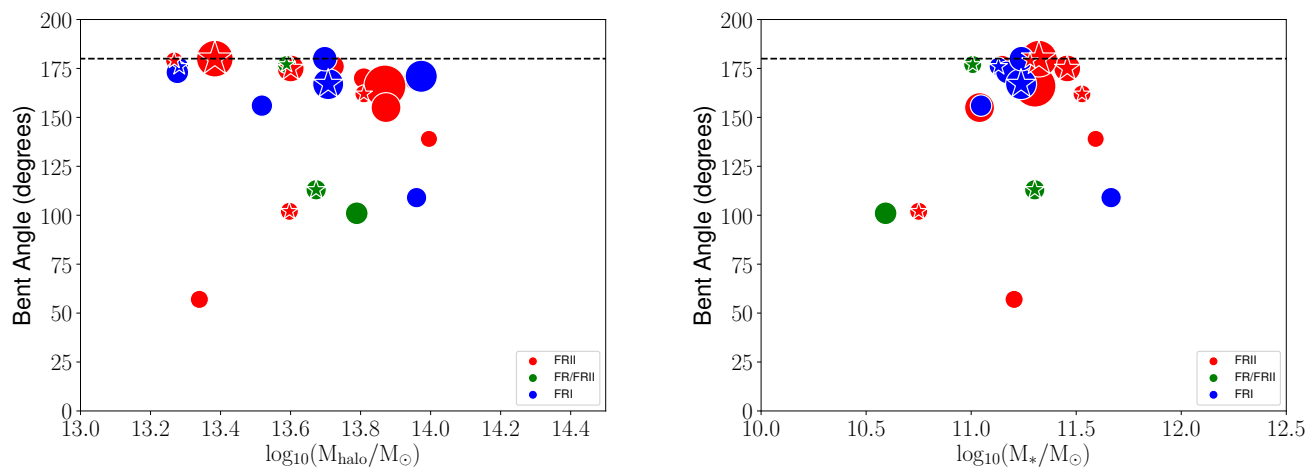

Figure 6. Bent angle in degrees for FRIIs (red), FRI/FRIIs (green), and FRIs (blue) as a function of the mass $M_{200}$ of the X-ray galaxy group [11,19] on the (left), and as a function of the stellar mass of the host $M_{*}$ on the (right). The horizontal dashed line marks a straight radio source where the jets or lobes form $180^{\circ}$. Symbol size is proportional to the $r / r_{200}$ ratio shown in Figure 5 , where larger symbols show objects farther away from the X-ray group center. In all panels, stars denote the brightest group galaxy (BGG). 


\subsection{Comparison to Simulations}

Vazza et al. [15] simulated the evolution of relativistic electrons injected into the ICM by radio galaxies, producing fully cosmological simulations of large-scale structures with ENZO and generating a template of radio galaxies by injecting seeds of supermassive black holes at run-time. They linked the thermal feedback related to the growth of black holes to the additional release of magnetic energy from opposite directions, leading to realistic "radio lobe" structures. In post-processing, the simulation also tracks the injection, propagation, and spectral energy evolution of a pool of relativistic electrons within the lobe regions, as well as their subsequent re-acceleration process by turbulence and shock waves, using a Fokker-Planck method; see [15] for further details. In the two runs analyzed here, radio galaxies start at the redshift of 0.5 or 1.0 in two independent "twin" simulations of the same galaxy group. Due to the continuous growth in mass of the host cluster, the two radio galaxies are hence formed in a slightly different environment (i.e., hotter and more pressurized in the first case), and are characterized by a different mass accretion rate onto the supermassive black hole at the center of the radio galaxy (i.e., a factor $\sim 100$ lower in the first case). Given that the simulation is fully cosmological, jets expand and interact with the surrounding environment and at the same time are also subject to the relative motion with the intracluster medium, which exerts a ram pressure onto the radio galaxy atmosphere as the latter travels at a $\sim 300 \mathrm{~km} / \mathrm{s}$ speed relative to the center of mass of the cluster. Although simulated jets are generated in perfect alignment at the opposite sides of the black hole, they are, since the beginning subject, to interactions with the intracluster medium. Especially during the very first stages of propagation of both jets (as can be seen in Figure 7, when the source is poorly resolved due to the finite spatial resolution of the simulation ( $\approx 8 \mathrm{kpc}$ in this region), the local fluctuations of the magnetic field intensity and electron number density, due to shocks and turbulence, make the estimate of the radio emission peak rather uncertain and unstable.

Here we analyzed the evolution of each simulated source in terms of how it grows in size every 25 Myrs in relation to the angle formed between the lobes/jets. We traced the position of the highest brightness peak in the lobe compared to the core position. This is shown as red dotted lines in Figure 8. We also trace the BA formed between the two edges of the sources (brightest pixels on each side), shown by the black solid line. For each 25 Myr time step, we mark how the linear projected size of the source increases given time for reference ${ }^{2}$.

Comparison of our COSMOS data from Figure 8 to the ENZO-MHD simulated sources at $z=0.5$ and at $z=1$ suggests a good agreement within the scatter (see Table 3 ), except in the very first epochs after the injection of simulated radio lobes, which are likely to be affected by numerical effect (as discussed above). The median BA of our COSMOS objects at a median redshift of around one is 167.5 degrees. The most bent objects in our sample are at lowest redshifts. To compare our observational results to the simulations, we calculate the median BA at two redshift bins, $0.4<z<0.6$ and $0.9<z<1.1$, shown in Table 3 . For the $z \sim 1$ redshift bin, we find the observations $\left(\mathrm{BA}_{\text {med }}=174.0_{163.9}^{178.2}\right)$ and simulations $\left(\mathrm{BA}_{\text {med }}=173.8_{151.0}^{177.4}\right)$ agree, within the scatter in the values. For the $z \sim 0.5$ redshift bin, the observations $\left(\mathrm{BA}_{\text {med }}=160.0_{111.5}^{178}\right)$ show a higher average bent angle than the simulated source $\left(\mathrm{BA}_{\text {med }}=122.6_{113.5}^{131.9}\right)$, but the median BA for the simulated source is within the scatter of the observations. This discrepancy suggests some sources of uncertainty in the observations. These could be projection effects that are more pronounced at low redshifts, classification biases, or different density environments. Based on our analysis of BA vs. density fields, FRIIs and FRI/FRIIs lie, on average, in denser environments at $z \sim 0.5$ than at $z \sim 1$ (by a factor of 2). The FRIs are found, on average, in less dense environments throughout. The difference between the environments of FRI, FRII, and FRI/FRII could justify the large scatter in BA. 


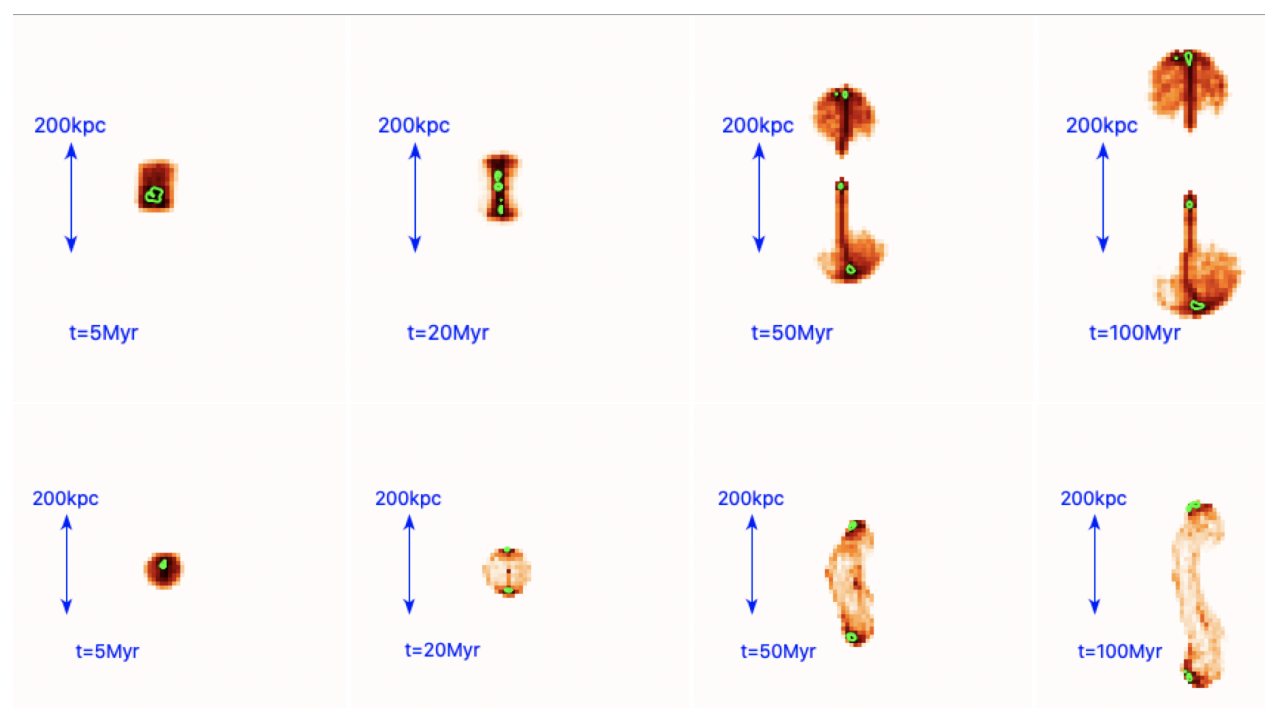

Figure 7. Map of radio emission at $1.4 \mathrm{GHz}$ for our simulated AGN for the case where the injection starts at $z=1.0$ (top) or at $z=0.5$ (bottom). The green contours mark the position of the brightness peaks in the image at different epochs, and the time refers to the initial injection epochs of both runs. In order to enhance the brightness substructures within jets at all epochs, the values of each map have been normalized in an arbitrary way.
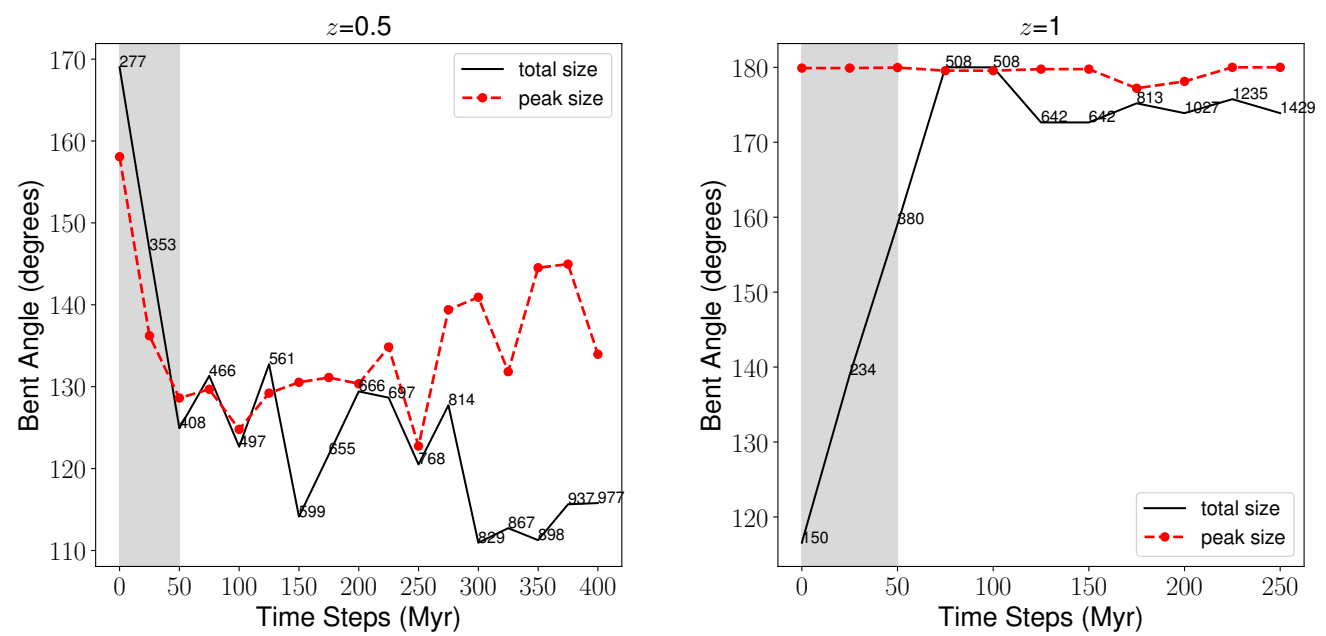

Figure 8. Simulated radio AGN at $1.4 \mathrm{GHz}$ from [15] at $z=0.5$ (left) and $z=1$ (right). We traced the evolution of the bent angle formed between source edges (black solid line) and source peak brightness (red dotted line) at both ends every 25 Myrs. The numbers show the linear projected size of the source in kpc. Time $t=0$ refers to the first frame of the simulation. The grey shaded region highlights the time range where the $\mathrm{BA}$ is uncertain.

For objects in COSMOS below a redshift of 0.5 , we have a median $\mathrm{BA}=167.5$ and error of the median \pm 17.5 degrees, which also falls in the range of values of the simulated source (Table 3). For objects within the range of $0.5<z<1.5$, we get a median BA of $167.0 \pm 22.2$ degrees. Finally, objects above a redshift of 1.5, beyond what our large-scale environments in COSMOS cover (apart from the density fields) and beyond the Vazza et al. simulations, have median BA $=168.0_{139.5}^{175.0}$ degrees. The difference in bent angle between the low- and high- $z$ simulated sources could be attributed to differences in the IGM density and the structure of the IGM itself. The median gas density value, as extracted from the simulation, is $2.15 \pm 0.20 \times 10^{-28} \mathrm{~g} / \mathrm{cm}^{3}$ at $z=0.5$ and $5.22 \pm 0.19 \times 10^{-29} \mathrm{~g} / \mathrm{cm}^{3}$ at $z=1$, an order of magnitude less. Additionally, the IGM at lower $z$ has a longer depth because the host cluster has a larger mass and its atmosphere is more extended, and thus the path 
the radio source has to pass is longer at lower redshifts. In other words, the lower the redshift, the wider the IGM distribution would be, which can promote more interaction and bending of the lobes.

Table 3. Median bent angle BA in different redshift bins within COSMOS and comparison to the ENZO-MHD simulated sources [15]. The BA of the simulated sources is traced over time.

\begin{tabular}{|c|c|c|c|c|c|}
\hline \multicolumn{3}{|c|}{ Observations } & \multicolumn{3}{|c|}{ Simulation } \\
\hline Redshift (z) & $N$ & Bent angle $84 \%$ (deg.) & $z_{\text {sim }}$ & $N$ & Bent angle $e_{16 \%}^{84 \%}$ (deg.) \\
\hline $0.4<z<0.6$ & 9 & $160.0_{111.5}^{178.6}$ & 0.5 & 1 & $122.6_{113.5}^{131.9}$ \\
\hline $0.9<z<1.1$ & 15 & $174.0_{163.9}^{178.2}$ & 1 & 1 & $173.8_{151.0}^{177.4}$ \\
\hline$z_{\text {med }} \sim 0.9$ & 108 & $167.5_{130.0}^{179.0}$ & & & \\
\hline$z<0.5$ & 88 & $167.5_{112.8}^{179.0}$ & & & \\
\hline $0.5<z<1.5$ & 58 & $169.5_{141.8}^{179.0}$ & & & \\
\hline $1.5<z<3$ & 20 & $168.0_{139.5}^{175.0}$ & & & \\
\hline
\end{tabular}

\section{Discussion}

Our analysis of the projected bent angle of $3 \mathrm{GHz}$ COSMOS FR-type radio sources gives a wide range of values, from very bent sources with $\mathrm{BA} \sim 40$ degrees to almost straight double-sided FR radio structures. In the context of galaxy evolution and AGN nature, we find it interesting that there are no obvious correlations with the large-scale environment and bent angle of extended radio AGN.

Within X-ray galaxy group environments (Figure 5), we find only 24 out of the 75 FRs, at the same redshift range and sky area coverage. This finding is related to the limitations of the current $X$-ray surveys within COSMOS [11] to detect galaxy groups with masses below $1.5(1+z) \times 10^{13} M_{\odot}[20]$, and bent FRs outside galaxy groups can be used to identify these. We only find 2 out of the $13(15 \%)$ straight FRs (BA = 180 degrees) inside the X-ray groups, which could be either because there are limitations to the current $X$-ray observations in COSMOS, or because straight FRs do not prefer $X$-ray group environments. It could also mean that the straight radio sources did not have time to interact with the medium and get their jets bent. An improvement over the COSMOS X-ray observations may shed light on this.

Regarding the density environments probing the large-scale structure in COSMOS, most of the bent FRs in our sample are found at surface densities of $1 / \mathrm{Mpc}^{2}$ (Figure 3), with a handful above densities of $10 / \mathrm{Mpc}^{2}$. The mild relation we find between FR type and BA is interesting and puzzling at the same time, given that FRIs, which are on average slightly more bent than FRIIs, are found in less dense environments than the other FR-types at $3 \mathrm{GHz}$ VLA-COSMOS. The latter result is also reported in [16], showing that FRIs reside in regions where environmental density, the number of galaxies per $\mathrm{Mpc}^{2}$, is lower than the other FR types. The smaller bent angles of FRIs could be caused by either movement of the jets through a denser ambient medium or movement of the objects through the ICM, in which case ram pressure bends the jets backwards; e.g., [4]. Given the large scatter of BA values in the FRI sample and given that the average environmental density of FRIs is lower than FRIIs with an overlap in the distributions, we believe that this is an object-to-object investigation. The bent angle is likely affected by more than one parameter.

The statistics given by the cosmic web probes (Figure 4) are small and the uncertainty is too large for a robust result. There is a mild trend for more bent FRs to lie in filaments. FRIs associated with satellite hosts are more bent than FRI/FRIIs and FRIIs. The same is seen if the host is a central galaxy, but the number of objects is small and the scatter large. Bent radio sources located several Mpc from cluster centers, in filaments, which have been reported before [21] and can be used to probe inter-filament density $\left(\sim 10^{-4}-10^{-6} \mathrm{~cm}^{-3}\right.$; see also [22]), which is less dense than what is expected for the gas in 
clusters. We understand that the interplay between hosts associated with FRIs plays a role in shaping their radio structures. If these hosts are satellite galaxies, which are less dynamically stable, it would explain the reason for distortions in the radio structure.

To investigate how the BA values of the FRs in our sample compare to those in other well-studied samples, we take the radio galaxy zoo project, RGZ [7], which studied 4304 bent radio galaxies inside clusters. They found that within the $\sim 1 \mathrm{Mpc}$ of the cluster, galaxies not associated with the brightest cluster galaxy, non-BCGs, are statistically more bent in high-mass clusters than in low-mass clusters. Contrary to that, we do not see a difference in the bent angle of non-BGG in relation to the group mass. Additionally, we do not find a difference between non-BGGs and BGGs in the inner $1 \mathrm{Mpc}$ (Figure 6). Furthermore, we do not find a difference in BA between non-BGGs and BGGs as we move closer to the group center (see Figure 5). This can also be seen in the left panel of Figure 6, where the size of the symbol increases with the distance from the group center.

The origin of such discrepancies between our study and the RGZ study might be a result of different selection criteria. Firstly, the total number of sources in our sample is $\sim 2 \%$ of that of the RGZ sample. Secondly, while our sample includes galaxy group masses in the range $M_{200 c}=8 \times 10^{12}-3 \times 10^{14} M_{\odot}$, the RGZ sample includes cluster masses $M_{500 c}=5-30 \times 10^{14} M_{\odot}$. To compare the COSMOS galaxy groups' halo masses to the RGZ cluster halo masses, we calculated the concentration and converted $M_{200 c}$ to $M_{500 c}$ using the code Colossus [23]. The parameters we used in their code are Planck15 cosmology and median redshift of COSMOS of 0.9 . We obtained $M_{500 c}=5 \times 10^{12}-2 \times 10^{14} M_{\odot}$. COSMOS is probing galaxy groups with smaller halo masses than probed by RGZ, and these groups are not dynamically relaxed as of yet [16]. The latter is related to the method being used to identify galaxies that belong in low-mass groups [11]. A low overdensity radius is used, which leads to finding loose groups that are not yet virialised. This could explain the difference in the results between the two studies regarding the BA of BGGs and non-BGGs.

In flux-density limited samples, we preferentially observe the brightest, and youngest [24], sources at higher redshifts. This is known as the youth-redshift degeneracy. Smaller sources at higher redshifts can have greater or lesser disruptions to their radio structure depending on how dense the ambient medium is $[25,26]$. Our observational result that lower-redshift FRs are, on average, more bent than higher-redshift FRs suggests that their energy deposit on the ambient medium is not enough to sustain the straight radio structure. Although our study is affected in places by small-number statistics (a K-S test between $z \sim 0.5$ and $z \sim 1$ bins (as in Table 3) gives a K-S statistic $=0.49$ and $p$-value $=0.21$, suggesting we cannot reject the null hypothesis that the distributions are similar) and possible projection effects, our results are in line with the ENZO-MHD simulations [15] presented here. Thus, there seems to be another important parameter that defines the median BA of radio sources, revealed by the comparison to the simulations: the redshift evolution of the IGM, which is well reproduced by the observational data. The IGM density at lower redshift is higher and has longer depth than at the higher redshift, which leaves more room for jet interactions. Thus, the radio jets become more perturbed and bent as they move across the IGM.

From the comparison to the simulations [15], we infer that the setup of the ENZOMHD simulations can mimic real sources. This is more obvious at the higher-redshift bin than the lower when compared to our sample of COSMOS FRs. What we see in the FRs in COSMOS is a snapshot of their evolution. The large scatter in our data compared to the simulated values represents the complexity of studying these objects in relation to their environment, as well as how difficult it is to understand the reason for the bent radio structure. We also see them at different stages of their evolution. We believe this is a case-to-case problem and needs to be approached in a multi-parameter manner. This will be the topic of a future study. 


\section{Conclusions}

We presented the analysis of the bent radio structure of FR-type radio AGN from the COSMOS field using the $3 \mathrm{GHz}$ VLA-COSMOS data [16,27]. To parameterize the complexity, we use measurements of the bent angle [16], the angle of the highest brightness peaks in the lobes/jets formed to each other. Our sample of 108 FRs has a median BA of $167.5_{-37.5}^{+11.5}$ degrees at a median redshift of 0.9 . We investigate the relation of the BA with several large-scale environmental probes within COSMOS (X-ray galaxy groups, density fields, and cosmic web). This study covers, for the first time, galaxy group halo masses $M_{200 c}=8 \times 10^{12}-3 \times 10^{14} M_{\odot}$, and the redshift range $0.08<z<3$ and scales from a few kpc up to $500 \mathrm{Mpc}$. Finally, we compare our observational BA values to the ones derived from the ENZO-MHD simulations of two radio sources at $z=0.5$ and at $z=1$ [15].

Our results are summarized as follows:

1. We do not find a significant correlation between BA and FR type.

2. FRIs extend to smaller BAs (i.e., they are more bent) than FRIIs and FRI/FRIIs.

3. We do not find a correlation between BA and large-scale environments or galaxy type.

4. We do not find a significant correlation between the BA of FR radio sources and their host X-ray galaxy group properties. More straight sources with higher group temperatures lie further away from the group center, while the BA of radio galaxies closer to the X-ray group center and with low temperature shows a large scatter.

5. The difference with large studies such as RGZ can be attributed to the fact that COSMOS is probing lower-mass halos and galaxy groups, which are not yet dynamically relaxed.

6. FRIs lie, on average, in less dense environments.

7. We do not find objects with $\mathrm{BA}<130$ degrees at densities below $<0.1 \mathrm{Mpc}^{-2}$.

8. The redshift evolution of BA in the simulations from $z=1$ to $z=0.5$ is reproduced by the observational data within the scatter.

Investigating relations to the large-scale environment is interesting in the COSMOS field and not intuitive given the fact we observe the faint radio universe at high sensitivity $(2.3 \mu \mathrm{Jy} / \mathrm{beam})$ and high resolution $\left(0^{\prime \prime} .75\right)$. We do not find significant trends with largescale environments within COSMOS. Comparison to simulations suggests a link between BA and redshift evolution. More bent sources at lower redshift can be explained with higher density and larger depth of the ambient medium, giving space to FRs to interact more. The larger scatter in BA at lower redshifts in our observational data could be explained by the difference between FRI, FRII, and FRI/FRII environments, as probed by the density fields in COSMOS.

We conclude that the dominant mechanism affecting the radio structures of FRs could be the IGM evolution with redshift, with higher density and greater depth at lower redshifts, allowing for more jet interactions, and not the large-scale environment. Our study, although limited in places due to small-number statistics, demonstrates the benefit of multiwavelength data at a very well-studied field like the COSMOS field. Future radio surveys in the SKA era, with increased sensitivity and resolution beyond current capabilities, can benefit from studies like this one and perform better statistics on extended radio AGN in order to identify the reasons behind distorted radio structures. Nevertheless, we believe that the parameter that is responsible for bent radio AGN is not only one parameter but can be a combination of parameters, from smaller scales within the host galaxy to larger scales outside the host, which we will investigate with future studies.

Author Contributions: Conceptualization, E.V.; methodology, E.V.; software, E.V.; validation, E.V.; formal analysis, E.V.; investigation, E.V.; resources, E.V., F.V., E.F.J.-A., G.G., A.F. and D.W.; data curation, E.V.; writing-original draft preparation, E.V.; writing-review and editing, E.V., F.V., E.F.J.-A., G.G., A.F. and D.W.; visualization, E.V.; project administration, E.V.; funding acquisition, E.V., F.V. All authors have read and agreed to the published version of the manuscript.

Funding: The simulations were funded by the European Union's Horizon 2020 program under the ERC Starting Grant "MAGCOW", no. 714196. D.W. is funded by the Deutsche Forschungsgemeinschaft (DFG, German Research Foundation)-441694982. 
Institutional Review Board Statement: Not applicable.

Informed Consent Statement: Not applicable.

Data Availability Statement: Data available at Vardoulaki et al. (2021); Scoville et al. (2013), and Darvish et al. (2017).

Acknowledgments: This study was presented by EV at the virtual conference "A new window on the radio emission from galaxies, clusters and cosmic web" on 10 March 2021, during the COVID-19 pandemic. We would like to thank the three anonymous referees for their very useful comments that improved the manuscript. EV acknowledges support by the Carl Zeiss Stiftung with the project code KODAR. The authors gratefully acknowledge the Gauss Centre for Supercomputing e.V. (www.gauss-centre.eu) for supporting this project by providing computing time through the John von Neumann Institute for Computing (NIC) on the GCS Supercomputer JUWELS at Jülich Supercomputing Centre (JSC), under project "radgalicm". FV acknowledges financial support from the European Union's Horizon 2020 program under the ERC Starting Grant "MAGCOW", no. 714196. D.W. is funded by the Deutsche Forschungsgemeinschaft (DFG, German Research Foundation)441694982 .

Conflicts of Interest: The authors declare no conflict of interest.

\section{Notes}

Data are publicly available at http:/ / irsa.ipac.caltech.edu/data/COSMOS/ancillary/densities/.

Videos of the two simulations can be found at https://vimeo.com/490397871 and https://vimeo.com/490399056.

\section{References}

1. Miley, G.K.; Perola, G.C.; van der Kruit, P.C.; van der Laan, H. Active Galaxies with Radio Trails in Clusters. Nature 1972, 237, 269-272. [CrossRef]

2. Owen, F.N.; Rudnick, L. Radio sources with wide-angle tails in Abell clusters of galaxies. Astrophys. J. Lett. 1976, 205, L1-L4. [CrossRef]

3. Burns, J.O. The Radio Properties of cD Galaxies in Abell Clusters. I. an X-ray Selected Sample. Astron. J. 1990, 99, 14. [CrossRef]

4. Smolčić, V.; Schinnerer, E.; Finoguenov, A.; Sakelliou, I.; Carilli, C.L.; Botzler, C.S.; Brusa, M.; Scoville, N.; Ajiki, M.; Capak, P.; et al. A Wide-Angle Tail Radio Galaxy in the COSMOS Field: Evidence for Cluster Formation. Astrophys. J. Suppl. Ser. 2007, 172, 295-313.

5. Hardcastle, M.J.; Sakelliou, I.; Worrall, D.M. A Chandra and XMM-Newton study of the wide-angle tail radio galaxy 3C465. Mon. Not. R. Astron. Soc. 2005, 359, 1007-1021.

6. Filipovic, M.D.; Crawford, E.J.; Jones, P.A.; White, G.L. Radio-Continuum Jets Around the Peculiar Galaxy Pair ESO $295-I G 022$. Serbian Astron. J. 2010, 181, 31-37.

7. Garon, A.F.; Rudnick, L.; Wong, O.I.; Jones, T.W.; Kim, J.A.; Andernach, H.; Shabala, S.S.; Kapińska, A.D.; Norris, R.P.; de Gasperin, F.; et al. Radio Galaxy Zoo: The Distortion of Radio Galaxies by Galaxy Clusters. Astron. J. 2019, 157, 126,

8. Golden-Marx, E.; Blanton, E.L.; Paterno-Mahler, R.; Brodwin, M.; Ashby, M.L.N.; Moravec, E.; Shen, L.; Lemaux, B.C.; Lubin, L.M.; Gal, R.R.; et al. The High-redshift Clusters Occupied by Bent Radio AGN (COBRA) Survey: Radio Source Properties. Astrophys. J. 2021, 907, 65,

9. O'Brien, A.N.; Norris, R.P.; Tothill, N.F.H.; Filipović, M.D. The spatial correlation of bent-tail galaxies and galaxy clusters. Mon. Not. R. Astron. Soc. 2018, 481, 5247-5262.

10. Scoville, N. The Cosmic Evolution Survey: COSMOS. From Z-Machines to ALMA: (Sub)Millimeter Spectroscopy of Galaxies; Baker, A.J., Glenn, J., Harris, A.I., Mangum, J.G., Yun, M.S., Eds.; Astronomical Society of the Pacific Conference Series; Astronomical Society of the Pacific: San Francisco, CA, USA, 2007; Volume 375, p. 166.

11. Gozaliasl, G.; Finoguenov, A.; Tanaka, M.; Dolag, K.; Montanari, F.; Kirkpatrick, C.C.; Vardoulaki, E.; Khosroshahi, H.G.; Salvato, M.; Laigle, C.; et al. Chandra centres for COSMOS X-ray galaxy groups: Differences in stellar properties between central dominant and offset brightest group galaxies. Mon. Not. R. Astron. Soc. 2019, 483, 3545-3565.

12. Scoville, N.; Arnouts, S.; Aussel, H.; Benson, A.; Bongiorno, A.; Bundy, K.; Calvo, M.A.A.; Capak, P.; Carollo, M.; Civano, F.; et al. Evolution of Galaxies and Their Environments at $\mathrm{z}=$ 0.1-3 in COSMOS. Astrophys. J. Suppl. Ser. 2013, 206, 3,

13. Darvish, B.; Mobasher, B.; Sobral, D.; Scoville, N.; Aragon-Calvo, M. A Comparative Study of Density Field Estimation for Galaxies: New Insights into the Evolution of Galaxies with Environment in COSMOS out to z 3. Astrophys. J. 2015, 805, 121,

14. Darvish, B.; Mobasher, B.; Martin, D.C.; Sobral, D.; Scoville, N.; Stroe, A.; Hemmati, S.; Kartaltepe, J. Cosmic Web of Galaxies in the COSMOS Field: Public Catalog and Different Quenching for Centrals and Satellites. Astrophys. J. 2017, 837, 16,

15. Vazza, F.; Wittor, D.; Brunetti, G.; Brüggen, M. Simulating the transport of relativistic electrons and magnetic fields injected by radio galaxies in the intracluster medium. arXiv 2021, arXiv:2102.04193. 
16. Vardoulaki, E.; Jiménez Andrade, E.F.; Delvecchio, I.; Smolčić, V.; Schinnerer, E.; Sargent, M.T.; Gozaliasl, G.; Finoguenov, A.; Bondi, M.; Zamorani, G.; et al. FR-type radio sources at $3 \mathrm{GHz}$ VLA-COSMOS: Relation to physical properties and large-scale environment. Astron. Astrophys. 2021, 648, A102. [CrossRef]

17. Fanaroff, B.L.; Riley, J.M. The morphology of extragalactic radio sources of high and low luminosity. Mon. Not. R. Astron. Soc. 1974, 167, 31P-36P. [CrossRef]

18. Laigle, C.; McCracken, H.J.; Ilbert, O.; Hsieh, B.C.; Davidzon, I.; Capak, P.; Hasinger, G.; Silverman, J.D.; Pichon, C.; Coupon, J. ; et al. The COSMOS2015 Catalog: Exploring the $1<\mathrm{z}<6$ Universe with Half a Million Galaxies Astrophys. J. 2016, 224, 24. [CrossRef]

19. Vardoulaki, E.; Gozaliasl, G.; Finoguenov, A.; Jiménez-Andrade, E.F.; COSMOS Team. The $\mathrm{M}_{*}-\mathrm{M}_{\text {halo }}$ Relation at $0.08<\mathrm{z}<1.53$ in COSMOS: The Role of Active Galactic Nucleus Radio-mode Feedback. Res. Notes Am. Astron. Soc. 2021, 5, 89. [CrossRef]

20. Vardoulaki, E.; Jiménez Andrade, E.F.; Karim, A.; Novak, M.; Leslie, S.K.; Tisanić, K.; Smolčić, V.; Schinnerer, E.; Sargent, M.T.; Bondi, M.; et al. A closer look at the deep radio sky: Multi-component radio sources at $3 \mathrm{GHz}$ VLA-COSMOS. Astron. Astrophys. 2019, 627, A142. [CrossRef]

21. Edwards, L.O.V.; Fadda, D.; Frayer, D.T. The First Bent Double Lobe Radio Source in a Known Cluster Filament: Constraints on the Intrafilament Medium. Astrophys. J. Lett. 2010, 724, L143-L147. [CrossRef]

22. Planck Collaboration.; Ade, P.A.R.; Aghanim, N.; Arnaud, M.; Ashdown, M.; Atrio-Barandela, F.; Aumont, J.; Baccigalupi, C.; Balbi, A.; Banday, A.J.; et al. Planck intermediate results. VIII. Filaments between interacting clusters. Astron. Astrophys. 2013, 550, A134. [CrossRef]

23. Diemer, B. COLOSSUS: A Python Toolkit for Cosmology, Large-scale Structure, and Dark Matter Halos. Astrophys. J. Suppl. Ser. 2018, 239, 35. [CrossRef]

24. Blundell, K.M.; Rawlings, S. The inevitable youthfulness of known high-redshift radio galaxies. Nature 1999, 399, 330-332. [CrossRef]

25. Kapahi, V.K.; Kulkarni, V.K.; Subrahmanya, C.R. On the interpretation of the observed angular-size-flux-density relation for extragalactic radio sources. J. Astrophys. Astron. 1987, 8, 33-50. [CrossRef]

26. Barthel, P.D.; Miley, G.K. Evolution of radio structure in quasars: A new probe of protogalaxies? Nature 1988, 333, 319-325. [CrossRef]

27. Smolčić, V.; Novak, M.; Bondi, M.; Ciliegi, P.; Mooley, K.P.; Schinnerer, E.; Zamorani, G.; Navarrete, F.; Bourke, S.; Karim, A.; et al. The VLA-COSMOS 3 GHz Large Project: Continuum data and source catalog release. Astron. Astrophys. 2017, 602, A1. [CrossRef] 